\title{
Überblick über die Entwicklung des ägyptischen Staatsrechts seit den 70er Jahren
}

\author{
Von Omaia Elwan
}

\section{Einleitung1}

Das ägyptische Rechtssystem hat seit Anfang der 70er Jahre bedeutende Neuerungen erfahren. Solche finden sich vor allem im

- Verfassungs- und Staatsrecht,

- Verwaltungsrecht,

- Zivilrecht (Familien-, Raummiet- und Agrarrecht),

- Handels- und Wirtschaftsrecht (Gesellschafts-, Handelsvertretungs- und Handelsregisterrecht, das Recht über die arabischen und ausländischen Kapitalinvestitionen, das Recht der Holdingorganisationen des öffentlichen Sektors und dessen Gesellschaften),

- Arbeitsrecht.

Im folgenden werden nur die Neuerungen in den zwei erstgenannten Rechtsbereichen dargestellt, auf die Entwicklung im Zivil-, Handels-, Wirtschafts- und Arbeitsrecht wird an anderer Stelle eingegangen.

\section{Hauptmerkmale der Verfassung von 1971}

Die Verfassung von 19712 weist, vor allem vor ihrer Änderung im Mai 1980, neben den meisten Hauptzügen ihrer Vorgängerin von 1964 folgende Merkmale auf:

1) Sie kann nur durch ein besonders erschwertes Verfahren geändert werden (eingeschränkter Kreis der zum Änderungsantrag Berechtigten, Zustimmung zur Änderung

1 Aus drucktechnischen Gründen wird hier auf eine buchstabengetreue Transkription der arabischen Wörter verzichtet. Es wird die nächstgelegene deutsche Entsprechung angewandt.

2 Vgl. Mustafa A. Fahmi, al-Qanun ad-dusturi, Alexandria 1984, S. 166 ff., Sulayman M. atTammawi, an-Nizam ad-dusturi al-misri, Magallat al-'Ulum al-Idariya, 27. Jg. (Dez. 1985), S. $7 \mathrm{ff}$. (60 ff.); Ahmad H.Nagm, Dirasa naqdiya li-dustur sanat 1971, ebenda. S. 156 ff.; S'ad 'Asfur, anNizam ad-dusturi al-misri, Alexandria 1980, S. 12 ff. 
durch qualifizierte Mehrheit der Volksversammlung, anschließende Zustimmung durch Volksbefragung: Art. 189).

2) Die Volksbefragung3 bleibt ein Mittel der Entscheidungsfindung, das in bestimmten Fällen sogar zwingend vorgesehen ist (z.B. bei Verfassungsänderungen gemäß Art. 189, Bestätigung der vom Staatspräsidenten getroffenen Eilmaßnahmen gemäß Art. 744; Wahl des von der Volksversammlung vorgeschlagenen Kandidaten zum Amt des Präsidenten gemäß Art. 76; Auflösung der Volksversammlung gemäß Art. 136).

3) Die allgemeinen klassischen Rechte und Pflichten der Bürger sind - als Antwort auf frühere Verstöße und Exzesse - deutlicher ausgebildet. So ist die Liste der sozialen und wirtschaftlichen Rechte, der allgemeinen Freiheiten und Pflichten der Bürger vollständiger als bisher (Art. 40-62). Verstöße dagegen (Schutz der privaten Lebenssphäre der Bürger sowie anderer verfassungsmäßig bzw. gesetzlich garantierter allgemeiner Freiheiten und Rechte) sind strafbare Handlungen, deren zivil- und strafrechtliche Verfolgung unver jährbar ist. Für eine gerechte Entschädigung des Opfers hat der Staat aufzukommen (Art. 56)5.

3 Kritisch zur Einführung der Volksbefragung in der Verfassung und zu den Gefahren von deren Mißbrauch in einem Land, dessen Bevölkerung einen hohen Prozentsatz an Analphabeten (70\%) aufweist und der folglich die politische Reife fehlt, vgl. Wahid $R^{\prime}$ afat, al-Wad' al-chas li-ra'is addawla bain sa'ir al-mu'assassat ad-dusturiya, Magallat al-Qanun Wa-l-Igtisad, Sondemummer (100-jähriges Jubiläum der Juristischen Fakultät) 1983, S. 566, 574 ff.; er greift vor allem die auf Anordnung von Sadat veranlaßten Volksbefragungen im Februar 1977, Mai 1978 und April 1979 an, die zum Erlaß von freiheitsbeschränkenden Gesetzen geführt hatten. Ebenso kritisch Magid alHulw, al-Istift'a asch-sch'abi, Alexandria 1983, S. $220 \mathrm{ff}$.

4 Kritisch zur dieser Bestimmung, die nicht die Kontrollsicherung des ihr vergleichbaren Art. 16 der franz. Verfassung (vorherige Konsultation des Premierministers, des Conseil Constitutionnel und der Präsidenten der beiden Kammem) enthält und folglich mißbraucht werden kann, wie dies sich im Februar 1977 und September 1981 gezeigt hat, vgl. Wagdy T. Ghubriyal, as-Sultat alistitha'iyal-ra'is al-gumhuriya, Alexandria, 1988, S. 166 ff.; $R$ 'afat (Fn. 3), S. 573 ff.; at-Tammawi (Fn. 2), $\mathrm{S} 9$ ff.; Husni Darwish Abd al-Hamid, al-Madda 74 mina al-dustur bain an-nazariya wa-1tatbiq, Magallat al-'Ulum al-idariya, 26. Jg. (Juni 1984), S. 101 ff. mit Erörterung der Rechtsnatur des Beschlusses des Staatspräsidenten, von Art. 74 der Verfassung Gebrauch zu machen (S. $117 \mathrm{ff}$.). Er setzt sich mit der Entscheidung des ägyptischen Verwaltungsgerichts (S. $122 \mathrm{ff}$.) in den Klagen Nr. 186, 187 am 22.12.1981, Nr. 1697 am 29. 6. 1982 und 2504 am 16.11.1982 (alle 36. Gerichtsjahr) auseinander. Das Gericht sah den Beschluß des Staatspräsidenten nicht als Hoheitsakt, sondem als Verwaltungsakt an und erklärte sich daher für dessen Kontrolle zuständig. Die nach Art. 74 erforderliche Grundvoraussetzung des Notstandes habe nach Auffassung des Gerichts in den oben angefochtenen Beschlüssen nicht vorgelegen.

5 Kritisch dazu Mahmud Mustafa, al-Mada 57 mina al-dustur, Magallat Al-Qanun Wal-Iqtisad, 1985, S. $1 \mathrm{ff}$. Er betrachtet diese Bestimmung als in der Formulierung zu weitgehend, zum einen hinsichtlich des Tatbestandes und zum zweiten wegen der Schärfe der Sanktion, da diese ausschließlich in einer Strafdrohung besteht. Zum Schutz des Privatlebens vor Fotografieren und 
4) Der Durchsetzung dieser Rechte und Pflichten nach den Prinzipien des Rechtsstaates (vor allem Unabhängigkeit und Immunität der richterlichen Gewalt, das Recht auf den gesetzlichen Richter und das Verbot, die gerichtliche Nachprüfbarkeit des Verwaltungshandelns durch Gesetze auszuschließen) ist ein ganzer Abschnitt (vierter Abschnitt: Art. 63-72) gewidmet.

5) Der "Sozialismus" durchzieht als Leitgedanke die Verfassung, vor allem vor ihrer Änderung 19806. Ziel der demokratisch-sozialistischen Wirtschaftsordnung soll jedoch nicht mehr die Auflösung der Klassen und deren Verschmelzung in einer Klasse sein, sondern die Verringerung der Einkommensdisparität, der Schutz des zulässigen Einkommens und die Zusicherung einer gerechten Verteilung der allgemeinen Lasten und Pflichten (Art. 4 n.F.) 7.

6) Der "sozialistische Generalankläger" (al-mud'ai al-ischtiraki) 8 ist eine Neuschöpfung der Verfassung von 1971. Er soll die Rechte des Volkes, die Sicherheit der Gesellschaft und ihres politischen Systems, die "sozialistischen Errungenschaften" und die Einhaltung des "sozialistischen Verhaltens" gewährleisten. Eine ausführliche Regelung des Amts des sozialistischen Generalanklägers ist in Art. 5-26 des Gesetzes Nr. 95/1980 über den "Schutz der Werte vor Schande"9 enthalten. Diesem Gesetz und der Verfassung läßt sich entnehmen, daß der sozialistische Generalanwalt nicht als Teil der Judikative gilt, sondern eher als eine Untersuchungs- und Klägerstelle, die der Kontrolle der Volksversammlung unterliegt. Er hat zwar beträchtliche, aber nicht alle in der Strafprozeßordnung aufgeführten Untersuchungszuständigkeiten und Befugnisse. So benötigt er zur Vornahme von Vorführung, Durchsuchung, Festnahme, Hausdurchsuchung, zum Abhören von Telefongesprächen und zur Kontrolle von Briefen einer Anordnung, die er im voraus bei dem im Wertegericht dazu ermächtigten Richter einzuholen hat.

Immerhin hat er nach dem Gesetz Nr. 34/1971 über "die Anordnung und Regelung von Sequestration sowie die Gewährleistung der Volkssicherheit"10 die Befugnis, bei begründetem Verdacht vorsorglich bis zur Gerichtsentscheidung ein Veräußerungs- und

Abhören sowie zur Änderung einiger Bestimmungen des StGB und der StPO betreffend die Freiheiten der Bürger wurde das Gesetz Nr. 37/1972 erlassen.

6 Vgl. Fahmi (Fn. 2), S. 178 ff.

7 Kritisch dazu Muhammad A. az-Zayyat, Misr ila ayna, 2. Aufl. Kairo 1986, S. 13 ff.

8 Vgl. dazu $\operatorname{Nagm}$ (Fn. 2), S. 178 ff.; al-'Adala, al-Mash al-igtima 'i asch-schamil lit-mugtam'a almisri, al-Markaz al-Qanwmi lil-Buhuth al-Igtima iya 1952-1980, Kairo 1985, S. 465 ff.; Kritisch zum Amt des Sozialistischen Generalanklägers Hussain K. al-Waqqad, al-Muhama, Mai/Juni 1988, S. $103 \mathrm{ff}$.

9 Dieses Gesetz wird von der Lehre stark kritisiert, vgl. dazu Mahmud Mustafa, Huquq al-insan fi '1igra'at al-gina'iya, in Magallat al-Qanun Wal-Iqtisad, Sondemummer 1983 (Fn. 3), S. $282 \mathrm{ff}$.

10 Kritisch zu diesem Gesetz, Mustafa (Fn. 9), S. 280 ff.; Nagm (Fn. 2), S. 185. 
Verwaltungsverbot des dem Beschuldigten, dessen Frau oder dessen Kindern gehörenden Vermögens anzuordnen und weiter, nach Einholung einer Anordnung des dazu ermächtigten Richters im Wertegericht, den Beschuldigten an einem sicheren Ort unter Arrest zu stellen bzw. ihm das Verlassen des Landes zu untersagen.

Desweiteren hat er nach dem Gesetz Nr. 95/1980 über "den Schutz der Werte vor Schande" die Befugnis zur Untersuchung und Anklage wegen politischer Verantwortlichkeit für die in diesem Gesetz aufgeführten Taten (siehe unten) vor dem neu geschaffenen "Wertegericht" (mahkamat al-qiyam). Da die Klagebefugnis zur Anordnung von Sequestration oder zur politischen Verantwortlichkeit auch einige Taten betrifft, die gleichzeitig die Voraussetzungen von strafbaren Handlungen erfüllen können, kann die Strafklage, falls der sozialistische Generalanwalt sich bereits mit der Sache befaßt hat, von der Staatsanwaltschaft erst auf seinen Antrag erhoben werden. Dadurch will man eine Verdoppelung der Verfolgungsverfahren, nämlich vor dem Wertegericht und vor dem Strafgericht, vermeiden.

Die beiden genannten, für den sozialistischen Generalankläger einschlägigen Gesetze bedürfen jedoch wegen widersprüchlicher, überflüssiger und unpräziser Regelungen einer Überarbeitung und Neukodifizierung.11

7) Das Einkammersystem bleibt de facto erhalten, da der durch Verfassungsänderung von 1980 geschaffene Konsultationsrat (maglis asch-schura) lediglich beratende Funktion hat.

\section{Verfassungsrechtliche Änderungen}

Die am 30.4.1980 durchgeführte Änderung der Verfassung von 1971 betraf Art. 1, 2, 4, 5 und 7712. Außerdem wurden der siebte und achte Abschnitt über den neugeschaffenen Konsultationsrat und die Anerkennung der Presse als unabhängige Volksgewalt hinzugefügt. Die Änderung des Art. 1 betraf seine Formulierung. Bei den anderen Änderungen handelte es sich um folgende materielle (inhaltliche) Regelungen:

11 Vgl. Nagm (Fn. 2), S. 183.

12 Vgl. Koen De Feyter, sub Egypt (April 1987), in Consitutiones Africae, Bd. II, Brüssel/Paris, S. $21,31 \mathrm{ff}$. 


\section{Stärkung der Scharia}

Die Grundsätze der islamischen Schar'ia sollen nunmehr die, d.h. die einzige und nicht wie ursprünglich - lediglich eine unter anderen Hauptquellen der Gesetzgebung sein, wodurch ihre Bedeutung erhöht wird (Art. 2)13. Die Änderung ist eine Folge der seit Anfang der 70er Jahre aufgekommenen und noch an Bedeutung zunehmenden Islamisierungsbewegung, die die Wiedereinsetzung der Schar'ia als einziges maßgebliches Rechtssystem verlangt14. Die Frage jedoch, welche Pflichten daraus für den Gesetzgeber entstehen, ist in der Lehre umstritten. Sollen die bestehenden Gesetze entsprechend geändert, deren Grundsätze lediglich bei Erlaß von neuen Gesetzen herangezogen oder sollen zumindest bei der Ubernahme von Normen aus fremden Rechtssystemen diese auf ihre Vereinbarkeit mit den Gesetzen der Schar'ia überprüft werden?

Der in der Verfassung vorgesehene, aber erst 1979 gebildete Hohe Verfassungsgerichtshof hat im Mai 1985 in zwei Fällen15 zur Frage der Vereinbarkeit sowohl von vor als auch nach der Verfassungsänderung von 1980 erlassenen Gesetzen gemäß Art. 2 in seiner neuen Fassung Stellung genommen. Im ersten Fall16 ging es um die Nichtigkeit des Art. 226 ägypt. ZGB, das 1949 erlassen wurde. Diese Vorschrift regelt die Frage der Verzugszinsen. Die Zinsnahme wird jedoch in der Schar'ia abgelehnt. Das Gericht bekannte sich in seinem Urteil zu folgenden Grundsätzen:

- Die Verzinsungsregelung in Art. 226 ägypt. ZGB ist nicht verfassungswidrig, obgleich die Zinsnahme in der Schar 'ia abgelehnt wird;

- Art. 2 der Verfassung, wonach die Grundsätze der islamischen Schar 'ia die Hauptquelle der Gesetzgebung darstellen, bedeutet keine unmittelbare Geltung des islamischen Rechts in Ägypten;

13 Vgl. A. Mahdi, De l'influence du droit musulman sur le droit positif en Egypte, Revue Juridique et Politique, 1984, S. $72 \mathrm{ff}$.

14 Vgl. Abd al-Hamid Mutawalli, asch-Schar'ia al-islamiya ka-masdr assasi lil-dustur, Alexandria (1975).

15 Dazu Elwan, Gesetzgebung und Rechtsprechung, in: Der Nahe und Mittlere Osten, hrsg. v. Steinbach/Robert, Opladen 1988, S. 237 ff.; 'Adel U. Scharif, Qad'a ad-dusturiya, Kairo 1988, S. $260 \mathrm{ff}$., kritisch Ahmad Z. asch-Schiyati, 'Adam ichtisas al-mahkama ad-dusturiya al-'ulya bi-ldaf'a , bi-'adam dusturiyat al-qawanin li-muchalafatiha lil-schar'ia al-islamiya, al-Muhama, Januar/Februar 1986, S. 42 ff., der die Zuständigkeit des Verfassungsgerichts für die Prüfung der Verfassungsmäßigkeit der Gesetze mit den Prinzipien der Schar'ia (Art. 2) vereint.

16 Beschluß vom 4.5.1985, Klage Nr. 20 1. Gerichtsjahr. Kritische Bemerkungen dazu: Ahmad K. Abu l-Magd, al-Ahram vom 2.7-1985, contra, Scharif(Fn. 15), S. 276. 
- die verfassungsrechtliche Prüfung von Rechtsvorschriften auf ihre Vereinbarkeit mit dem islamischen Recht beschränkt sich auf nach der Verfassungsänderung vom 22. Mai 1980 erlassene Gesetze;

- alle nach der Verfassungsänderung erlassenen Rechtsvorschriften müssen zur Vermeidung der Nichtigkeit mit den Prinzipien der islamischen Schar 'ia vereinbar sein;

- der Gesetzgeber ist durch die Verfassung aufgefordert, schrittweise das gesamte Rechtssystem mit den Prinzipien der islamischen Schar' ia in Einklang zu bringen.

Damit ist für diesen konkreten Fall eine mit der Schar'ia möglicherweise nicht vereinbare Regelung als verfassungsgemäß erklärt worden.

Im zweiten Fall wurde beantragt, das "Gesetz Nr. 44/1979 betreffend die Regelung einiger Fragen des Personalstatuts" für nichtig zu erklären, weil viele seiner Vorschriften gegen die Grundsätze der Schar 'ia verstoßen würden. Das Gericht erklärte zwar das genannte Gesetz für verfassungswidrig17, aber nicht aus dem dargelegten, sondern aus einem formellen verfassungsrechtlichen Grund. Die beanstandete Regelung war als Präsidialbeschluß erlassen worden. Nach Auffassung des Gerichts lagen dabei die Voraussetzungen des Art. 14718 der Verfassung nicht vor, der den Staatspräsidenten außerhalb der Sitzungsperiode der Volksversammlung zum Erlaß der angegriffenen Regelung in Form eines Präsidialbeschlusses berechtigt hätte.

Aus diesen beiden Fällen ergibt sich, daß eine absolute Vorrangigkeit der Schar'ia bisher in der ägyptischen Rechtsprechung nicht gegeben ist.

17 Beschluß vom 4.5.1985, Magmu'at al-ahkam as-sadira 'an al-mahkama ad-dusturiya al-'ulya, Bd. 3, S. 195; vgl. dazu Elwan (Fn. 15); Muhammad N. Kamel, Ichtisas al-mahkama ad-dusturiya al'ulya, Kairo 1989, S. $301 \mathrm{ff}$.

18 Vgl. dazu Democracy in Egypt: Problems and Prospects, hrsg. von Ali E. H. Dessouki, Cairo Papers (The American University) Vol. 1, Nr. 2, 2. Aufl. 1983, S. 8-26, 41-45; Tariq F. Chidr, Dawr al-ahzab as-siyasiya, Kairo 1986, S. 139 ff.; Mustafa K. as-Sayid, Taqyim tagribat t'adud alahzab (1976-1981), in Tagribat ad-dimuqratiya fi misr (Sammelwerk von IAli Hilal u.a.), 3. Aufl. Kairo 1985, S. 197-247; R. Glagow, Die Wiedereinführung des Parteiensystems in Ägypten, Orient Dez. 1976, S. 58 ff.; G. Krämer, Ägypten unter Mubarak, Baden-Baden 1986, S. 46 ff. 


\section{Zulassung des Mehrparteiensystems}

\section{a) Die Pluralisierung ab 1976}

Das politische System der Arabischen Republik Ägypten wird nunmehr auf der Vielfalt der Parteien beruhen, allerdings unter Beachtung der wesentlichen Grundsätze der ägyptischen Gesellschaft, wie sie in der Verfassung zum Ausdruck kommen (Art. 5). Status und Tätigkeit der politischen Parteien sind durch Gesetz zu regeln.

Die Parteien entstanden im Zuge der nach dem Tode Abdel Nassers eingeleiteten politischen Liberalisierung. Im Juli 1975 bildeten sich lose strukturierte Meinungsforen innerhalb der Arabischen Sozialistischen Union, der bis dahin in Ägpyten einzigen zugelassenen Einheitspartei. Von 40 angemeldeten Meinungsforen wurden drei zugelassen. Diese wurden Ende März 1976 in sogenannte Organisationen umgewandelt. Unmittelbar nach den Wahlen zur Volksversammlung, an denen sie teilnehmen durften, erklärte sie der Staatspräsident im November 1976 zu rechtmäßigen Parteien 19.

Am 2. Juli 1977 wurde das Gesetz Nr. 40 zur Regelung der politischen Parteien erlassen, das durch die Gesetze Nr. 33/1978, 36/1979, 144/1980, 36/1981 und 130/1981 geändert wurde20. Ferner wurde das Gesetz Nr. 38/1972 vom 28.09.1972 betreffend die Volksversammlung durch die Gesetze Nr. 23/1979, 144/1983 und 188/1986 geändert, um es an die neu geschaffene Lage nach der Institutionalisierung des Mehrparteiensystems anzupassen.

Art. 4 des Parteiengesetzes nennt neun Voraussetzungen zur Gründung einer Partei. Vor allem sind folgende Grundsätze zwingend zu beachten 21: die Grundsätze

- der islamischen Schar 'ia, die die Hauptquelle der Gesetzgebung darstellen;

- der Revolutionen vom 23.07.1952 und 15.05.1971;

- der nationalen Einheit, des sozialen Friedens, des sozialistischen Systems und der sozialistischen Errungenschaften.

Ferner darf eine Partei in ihrem Bekenntnis und Programm, in ihrer praktischen Tätigkeit und in der Wahl ihrer Funktionäre bzw. Mitglieder nicht gegen die Bestimmungen des Gesetzes Nr. 33/1978 über den "Schutz der inneren Front und des sozialen Friedens" verstoßen, u. a. nicht die Zugehörigkeit zu bestimmten Klassen, Konfessionen, Gruppen

19 Vgl. Chidr (Fn. 18), S. 140 ff., 'Asfur (Fn. 2), S. 42 ff.

20 Kritisch dazu 'Asfur (Fn. 2), S. 43 ff.; Fahmi (Fn. 2), S. 229; 'Abd al-Hamid Mutawalli, Nazarat fi anzimat al-hukm fi l-duwal an-Namiya, Alexandria 1985, S. 467.

21 'Asfur a.a.O. weist darauf hin, daß die Bewertung des Unterlassens eines Bescheides als Ablehnung und die besondere Zusammensetzung der Kammer des Hohen Verwaltungsgerichts erst durch das Gesetz Nr. 36/1979 eingeführt seien, welches die Gründung von neuen Parteien erschwert habe. 
und Regionen fordem, bzw. eine Rassen-, Herkunfts-, Religions- oder Glaubensdiskriminierung praktizieren.

Zur Gründung einer politischen Partei bedarf es einer Genehmigung. Der Antrag muß von mindestens 50 Gründungsmitgliedern beim Ausschuß für politische Parteien gestellt werden. Dieser Ausschuß besteht aus dem Präsidenten der Volksversammlung, den Ministern der Justiz und des Innern, dem Staatsminister für Fragen der Volksversammlung sowie aus drei parteilosen ehemaligen Präsidenten bzw. Vizepräsidenten von Gerichten. Wird über den Antrag innerhalb von drei Monaten nicht entschieden, so gilt er als abgelehnt. Gegen die Ablehnung des Antrags kann Rechtsmittel vor der Ersten Kammer des Hohen Verwaltungsgerichts eingelegt werden, deren Zusammensetzung allerdings durch eine gleiche Anzahl gewählter Mitglieder aus der Mitte der Abgeordneten der Volksversammlung erweitert wird22.

Im Vergleich zu westlichen Demokratien ist damit die Gründung von politischen Parteien vor allem durch zahlreiche erschwerende Gründungsvoraussetzungen sowie das Erfordemis einer Genehmigung eingeschränkt, die von einem Ausschuß aus nicht neutralen Mitgliedern erteilt werden muß23.

22 Vgl. as-Sayid (Fn. 18), S. 231 ff.; Fahmi (Fn. 2), S. 253 ff.; 'Asfur (Fn. 2), S. 58 ff., Mutawalli (Fn. 21), S. 398 ff. Die Aussichten für einen positiven Zulassungsbescheid des Parteienausschusses sind sehr gering. Die Zulassung wird fast nur durch einen Gerichtsbeschluß erreicht. Vgl. z.B. den Beschluß des Hohen Verwaltungsgerichts vom 25.6.1983 (Beschwerde Nr. 1202 26. Gerichtsjahr) betreffend die Umma Partei (unveröffentlicht, auszugsweise abgedruckt in Hidr (Fn. 18), S. 144 ff.) und den Beschluß vom 14.4.1990, der die Gründung dreier neuer Parteien (Misr al-Fatah, die Unionistische Demokratische Partei und die Grünen Ägyptens) zugelassen, aber die Aufhebung des ablehnenden Gründungsbescheids betreffend die Nassiritische Partei vom 18.12.1983 zurückgewiesen hat. Das Gerichts begründet letzteres dadurch, daß diese Partei von einem hegemonialen politischen System ausgehe und sich nicht zur Demokratie und zum Mehrparteiensystem bekenne. Vgl. dazu Ahmad Hussain, in al-Ahram al-Iqtisadi Nr. 1112 vom 7.5.1990, S. 32f; al-Hawadeth (London), Nr. 1750, vom 18.5.1990, S. 34, Alhayat Nr. 9959 vom 21./22.4.1990, S. 8. Schon vor diesem Beschluß hat der Verfassungsgerichtshof am 7.5.1988 den Antrag der Nassiritischen Partei, Art. 4 Abs. 7 des Gesetzes Nr. 40/1977 wegen Verstoßes gegen Art. 5 und 47 der Verfassung für verfassungswidrig zu erklären, stattgegeben. Nach der angefochtenen Bestimmung waren Parteigründer wegen Aufforderung zur Auflehnung gegen das mit Israel geschlossenen Camp-DavidAbkommen auszuschließen. Vgl. dazu at-Taqrir al-istratigi al-'arabi 1988, hrsg. von as-Sayyid Yassin, Kairo 1989, S. 474; Kamil (Fn. 17), S. 182f, sowie Fn. 3, S. 256 ff., Fn. 2 und S. 266.

$23 \mathrm{Vgl}$. Art. 87. Gemäß Art. 1 des Gesetzes Nr. 38/1972 i. F. des Gesetzes Nr. 114/1983 besteht die Volksversammlung aus 448 Mitgliedem. Kritisch zur Befugnis des Staatspräsidenten, bis 10 Mitglieder in der Volksversammlung zu emennen, und zum 50\%igen Anteil von Bauem und Arbeitem an den Sitzen vgl. 'Asfur (Fn. 2), S. 170, 179f, Nagm (Fn. 2), S. 170 ff., ebenso zum Sitzanteil Mutawalli (Fn. 20), S. 298 ff., a. Auffassung Fahmi (Fn. 2), S. 329f. Die Definition des Arbeiters und Bauem ist diffus und gestattet, $\mathrm{da} B$ sich manche ungerechtfertigterweise diesen Gruppen zurechnen, 'Asfur, a.a.O. S. 180. Zu dieser Definition vgl. at-Tammawi (Fn. 2), S. 73; P. Mirel, Le Parlement Egyptien, Revue Juridique et Politique 1980, S. 843, Fn. 6, der bemerkt, daß 


\section{b) Staatliche Eingriffe in das Mehrparteiensystem ab 1977}

Außer der Aufnahme des Mehrparteiensystems blieben die übrigen Regelungen der Verfassung von 1971, die auf ein Einparteiensystem zugeschnitten waren, unverändert. Demnach fehlt es an einer Regelung der Beziehungen des Staatspräsidenten zu den politischen Parteien: der Ministerpräsident und die Minister werden vom Staatspräsidenten ernannt; der Staatspräsident wird auf Vorschlag der Volksversammlung unmittelbar vom Volk gewählt. Dies führte zu Unklarheiten über die Spielregeln der politischen Arbeit und beließ sie in der Abhängigkeit von willkürlichen Entscheidungen des Staatspräsidenten, wie dies die Erfahrungen während der Amtszeit des Präsidenten Sadat gezeigt hat. Nachdem er das Mehrparteiensystem im Jahre 1976 zugelassen hatte, legte er der Arbeit der Oppositionsparteien kurz nach den durch einschneidende Wirtschaftsmaßnahmen ausgelösten Unruhen in der Bevölkerung gravierende Beschränkungen auf, da er bestimmte Oppositionsparteien für diese Unruhen verantwortlich machte. Er erließ dazu zahlreiche Gesetze mit unverkennbarem Ausnahmecharakter, von denen die Gesetze Nr. 2/1977 über den "Schutz der nationalen Einheit", Nr. 40/1977 über die politischen Parteien, Nr. 33/1978 über den "Schutz der inneren Front und den sozialen Frieden", Nr. 95/1980 über den "Schutz der Werte vor Schande" und Nr. 148/1980 über die "Pressegewalt" zu erwähnen sind. Sadat ging sogar so weit, 1979 während seiner Amtszeit eine eigene Partei zu gründen, die die bisherige Regierungspartei ablöste, wobei er den Vorsitz der neuen Partei sich selbst vorbehielt. Die in der Verfassung fehlende Regelung des Verhältnisses der politischen Parteien zu den anderen Organen erlaubte Sadat diese Kumulation von Ämtern. Im Verhältnis zwischen dem Amt als Staatspräsident und dem als Parteivorsitzendem hat sich Mubarak indessen für eine Fortsetzung der Ämterkumulation entschieden. Dies geschah gegen den Rat mancher Verfassungsrechtler, die sich für eine Trennung ausgesprochen hatten, um dem Präsidenten eine Neutralität zu gewähren, die ihm erlauben würde, fern von Parteiinteressen im nationalen Interesse zu handeln und sein politisches Schicksal nicht allzu sehr an das seiner Partei zu binden, da diese in einer Wahl auch die Mehrheit einbüßen könnte24. Die Stärkung der Regierungspartei durch die Ämterkumulation mag der Grund dafür sein, daß das neue Wahlgesetz für Oppostitionskandidaten so restriktiv ausgefallen ist, daß es zum zweiten Mal für verfassungswidrig erklärt wurde.

Nach der Ermordung Sadats schlug Präsident Mubarak zwar einen Entspannungskurs ein, die oben genannten Gesetze sind aber bis heute unverändert in Geltung geblieben. Dabei

die Bauem und Arbeiter trotz dem ihnen vorbehaltenen Sitzanteil in der Volksversammlung unterrepräsentiert seien.

24 Vgl. dazu Hassan Naf'a, al-Idara as-Siyassiya li-azmat at-tahawwul min nizam al-hizb al-wahid ila nizam t'adud al-ahzab, in al-Nizam as-Siyassi al-misri, hrsg. von 'Ali ad-Din Hilal, Kairo 1988, S. $26 \mathrm{ff}$., $54 \mathrm{ff}$. 
wird jedoch im Bereich der täglichen politischen Arbeit von der Anwendung dieser Gesetze, mit Ausnahme des Gesetzes über die Parteien, abgesehen.

Diese Haltung führte zu Widersprüchlichkeiten25. Auf der einen Seite wird den Anträgen auf die Gründung neuer Parteien unter Berufung auf das strenge Parteiengesetz nicht stattgegeben, auf der anderen Seite wurde toleriert, daß eine schon zugelassene Partei ihr Programm änderte und zu den Wahlen mit einem Wahlspruch wie "der Islam ist die Lösung" antrat, um den "Moslem-Brüdern", denen die Gründung einer dem Islam verpflichteten Partei verwehrt wird, zu gestatten, sich unter dem Deckmantel der Liste der genannten Partei als Wahlbewerber aufstellen zu lassen und Sitze in der Volksversammlung zu erhalten. Als zweite Widersprüchlichkeit ist zu nennen, daß Parteien sich zwar frei äußern dürfen - etwa in der Presse - , daß ihnen aber das Abhalten öffentlicher Versammlungen verwehrt wird.

Ein weiterer Widerspruch besteht darin, daß die Oppositionsparteien in der Tat unter der bestehenden Beschränkung der politischen Arbeit leiden, in ihren eigenen Reihen aber die demokratischen Regeln nicht praktizieren. Sie haben meistens autoritäre Führer der alten, durch die Revolution abgeschafften Parteien als Vorsitzende, und es fehlt ihnen an Statuten oder klaren internen Geschäftsordnungen.

Schließlich ist auf den Kontrast zwischen dem eingeleiteten Demokratisierungsprozeß und dem schwachen Interesse der Bevölkerung, sich daran zu beteiligen, hinzuweisen. Von den in die Wahlverzeichnisse eingetragenen Wählem, die weniger als $45 \%$ der tatsächlichen Anzahl der Wahlberechtigten ausmachen, nehmen tatsächlich weniger als $40 \%$ an den Wahlen teil. Dieses Desinteresse wird auf das Wesen der zugelassenen Parteien und deren Programme zurückgeführt. Denn einerseits ist die Regierungspartei durch Beschluß des Staatspräsidenten geschaffen worden, und es fehlt ihr an einer echten Basis in der Bevölkerung. Andererseits sind die Oppositionsparteien auch nicht mehr als Gruppen, die von der Persönlichkeit des jeweiligen Führers und von vagen Programmen geprägt sind, so daß sie keinen Rückhalt in den Reihen der Bevölkerung haben. Ferner wird die Masse der Bevölkerung wegen der außerordentlich kritischen Wirtschaftsverhältnisse des täglichen Lebens durch die Sorge bei der Suche nach ausreichendem Lebensunterhalt vom politischen Leben abgelenkt. An die Stelle des Staates, dessen Leistung als Wohlfahrtsstaat sich durch den Rückgang der Einnahmen und die Bevölkerungszunahme nach und nach vermindert, sind private Vereine, von denen ein Teil religiös orientiert ist, und Berufsverbände getreten, die zum Teil dessen Rolle übernommen haben26. Es bleibt abzuwarten, ob es gelingt, diese Vereine und Berufsverbände wegen ihres unmittelbaren Kontaktes zu den Bürgern für das

25 Ali Hilal, L'évolution politique de l'Egypte: pluralisme démocratique ou néo-autoritarisme?, in: Maghreb Machrek Nr. 127 (Januar-März 1990), S. 7 ff., 11 ff.

26 Hilal (Fn. 25), S. 13 ff. 
politische Leben zu interessieren, wobei ein Problem darin besteht, daß die Vereine - im Unterschied zu den Berufsverbänden - ihren jeweiligen Religionen verbunden sind, was im Falle ihres politischen Engagements zu Konfessionskonflikten führen könnte.

\section{Stärkung der Legislative}

Die Volksversammlung, der die Gesetzgebung zusteht, wird durch unmittelbare, allgemeine Wahlen gebildet. Sie besteht aus mindestens 350 Mitgliedern, von denen zumindest die Hälfte Bauern oder Arbeitnehmer sein müssen. Der Staatspräsident kann ferner bis zu 10 Mitglieder ernennen (Art. 87 der Verfassung)27. Die Emennung soll einerseits qualifizierten Personen, die sich den besonderen Bedingungen eines Wahlkampfes nicht unterziehen wollen, andererseits auch Parteien, deren Kandidaten über die Wahlen keine Mandate erhalten haben, den Zugang zur Volksversammlung öffnen.

Das Wahlsystem ist durch die Gesetze Nr. 38/1972 über die Volksversammlung und Nr. 73/1956 zur Regelung der Ausübung der politischen Rechte geregelt. Die genannten Gesetze wurden mehrmals geändert bzw. ergänzt, zuletzt durch Gesetz Nr. 188/1986. Dieses sieht eine Mehrheitswahl von Direktmandaten und eine Verhältniswahl nach Parteienlisten vor.

Die Aufstellung der Listen ist den zugelassenen Parteien vorbehalten und Restriktionen unterworfen. In den Wahllisten dürfen nur Mitglieder der Parteien aufgenommen werden. Demnach sind gemeinsame, mit anderen Parteien aufgestellte Listen unzulässig. Die Liste muß genau die Zahl von Kandidaten enthalten, die der für den Wahlkreis bestimmten Sitzzahl entspricht. Von den Wahlkandidaten müssen mindestens 50\% Bauern und Arbeiter sein. Der Wähler darf seine Stimme nur für eine Liste insgesamt und ohne jegliche Änderung abgeben. Es besteht außerdem eine 8\%-Sperrklausel. Die Sitzverteilung erfolgt entsprechend dem Anteil der Listen an den abgegebenen Stimmen.

Wahlberechtigt sind alle in das Wahlregister eingetragenen Staatsangehörigen, d.h. die Männer und Frauen, die das 18. Lebensjahr vollendet haben. Die diskriminierenden Regelungen, daß Frauen erst auf ihren Antrag in das Wahlregister eingetragen werden und ihnen in 30 bestimmten Wahlkreisen Sitze vorbehalten sind, wurden durch das Gesetz Nr. $188 / 1986$ aufgehoben.

Neben Mandaten, die durch Listenwahl nach dem Verhältnisprinzip indirekt gewählt werden, gibt es seit 1986 in den 48 neu eingeteilten Wahlkreisen solche, die nach dem

27 Vgl. zum Wahlsystem S'uad asch-Scharqawi Nuzum al-intichabat, Kairo 1984 S. 153 ff.; Chidr (Fn. 18), S. 313 ff.; at-Tammawi (Fn. 2), S. 70 ff. 
System der relativen Mehrheit direkt vergeben werden. Zur Vergabe des Direktmandats im ersten Wahlvorgang muß der Kandidat, auf den die meisten Stimmen entfallen, zumindest $20 \%$ der abgegebenen Stimmen erhalten haben.

Der Verfassungsgerichtshof hat am 19.5.1990 einige Bestimmungen des Gesetzes Nr. 188/1986 für verfassungswidrig erklärt, wie er dies schon am 16.5.1987 hinsichtlich des Gesetzes Nr. 114/198328 getan hat. Demnach seien zwar die im Jahre 1987 abgehaltenen Wahlen zur Volksversammlung nichtig, die von ihr beschlossenen Gesetze blieben aber gültig. Ein neues Wahlgesetz soll durch einen unabhängigen Ausschuß erstellt werden, da die jetzige Volksversammlung nach der Veröffentlichung der Entscheidung des Verfassungsgerichtshofes im Gesetzesblatt nicht handeln kann und aufgelöst werden soll29.

\section{Einsetzung eines Konsultationsrates (maglis asch-schura)}

Der Konsultationsrat ist im Rahmen der Verfassungsänderung von 1980 geschaffen worden. Seine Zusammensetzung und seine Aufgaben sind in den der Verfassung angefügten Artikeln 194 - 205 geregelt. Ausführliche Bestimmungen dazu sind in Gesetz Nr. 120/198030 enthalten, das von Gesetz Nr. 10/1989 abgelöst wurde.

28 Kamal Chalid, as-Sir'a m a tariyat al-qawanin, Kairo 1989, dem es gelungen ist, einige Bestimmungen dieses Gesetzes (Art. 5 bis, 6/1, 17/1) durch den Verfassungsgerichtshof am 16.05.1987 wegen der Beschränkung der Kandidatur zu Wahlen auf die in den Wahllisten der zugelassenen Parteien aufgeführten Personen für verfassungswidrig erklären zu lassen. Das Gericht sah in dieser Beschränkung eine Verletzung der Chancengleichheit, des Gleichheitsprinzips und des Wahlrechts (Art. 8, 40, 62 der Verfassung). Als die Regierung von der ungünstigen Vorlage erfuhr, die von dem dem Gericht zugeordneten und für die Prozeßvorbereitung zuständigen Ausschuß der "Commissaires" (hay at al-Mufawadin) zum genannten Gesetz dem Verfassungsgerichtshof eingereicht hatte, erließ sie das Gesetz Nr. 188 vom 31.12.1986, das mehrere Bestimmungen des beanstandeten Gesetzes Nr. 38/1972 änderte. Die Volksversammlung wurde im Anschluß an eine Volksbefragung aufgelöst, und neue Wahlen wurden am 6.4.1987 durchgeführt. Vgl. femer Muhammad M. Abu al-'Ainayn, Qanun al-intichab bi-l-qa ima al-nisbiya, al-Muhama, Nr. 7/8 (Sept./Okt. 1987, S. 57 ff.)

29 Vgl. die Tageszeitung Alhayat vom 22.5.1990, S. 1 u. 7; al-Ahram vom 1.6.1990, S. 1 u. 6. Halid (Fn. 25) hat auch diese Verfassungsklage erhoben, durch die er die Verfassungswidrigkeit der Art. 3 und 5 b des Gesetzes Nr. 188/1986 beanstandet hat. Vgl. dazu sowie zu dem dieser Klage vorausgehenden Beschluß des Verwaltungsgerichtshofes vom 31.3.1987 Abu al-'Ainayen (Fn. 25).In jedem der nach dem angefochtenen Gesetz Nr. 188/1986 neu eingeteilten 48 (bisher 176) Wahlbezirke (Art. 3) sollte es ein einziges Direktmandat neben mehreren Listenmandaten (Art. 5 bis) geben. Dadurch hatte ein Wähler, der keine Liste wählen wollte, nur die Möglichkeit, den Direktkandidaten zu wählen; wer eine Liste wählte, konnte je nach der Größe des Wahlkreises zusätzlich bis zu etwa 12 Kandidaten wählen. Das Verfassungsgericht sah in Art. 5 b des Gesetzes Nr. 38/1972 i.F. des Gesetzes Nr. 188/1986 einen Verstoß gegen Art. 40 und 62 der Verfassung.

30 Vgl. at-Tammawi (Fn. 2) S. 84 ff.; Ahmad H. Nagm, Nizam al-maglisayn, Magallat al-'Ulum alIdariya 28. Jg. (Dezember 1986), S. $111 \mathrm{ff}$. 
Der Konsultationsrat gilt nicht als eine zweite Kammer, da er keine legislativen Zuständigkeiten, sondem nur beratende Aufgaben hat. Ein Drittel seiner Mitglieder wird vom Staatspräsidenten emannt, und die übrigen werden durch unmittelbare, geheime und allgemeine Wahlen bestimmt. Das bisherige Listenwahlsystem ist durch Gesetz Nr. 10/198931 abgeschafft worden. Zur Wahl stehen nunmehr nur noch Direktmandate. Von den gewählten Mitgliedern müssen mindestens die Hälfte Arbeiter und Bauern sein.

Hauptaufgabe des Konsulationsrates ist es, Untersuchungen über bestimmte Fragen anzustellen und zweckdienliche Vorschläge zu machen. Diese Untersuchungen, die auf eigene Initiative oder auf Vorschlag des Staatspräsidenten unternommen werden, haben folgende Ziele:

- Sicherung der Grundsätze der Revolutionen von 1952 und 1971;

- Schutz der arbeitenden Volkskräfte, der sozialistischen Errungenschaften, der Grundlagen und Werte der Gesellschaft, der Grundrechte und Pflichten der Bürger sowie der Stärkung des sozialistischen Systems.

Außer dieser Hauptaufgabe nimmt der Konsultationsrat in folgenden Fällen Stellung:

- zu Vorschlägen zur Verfassungsänderung;

- zu Entwürfen zur Ergänzung der Verfassung;

- zum Entwurf des sozialen und wirtschaftlichen Entwicklungsplanes;

- zu Friedens- und Allianzabkommen sowie Ubereinkommen betreffend das Staatsterritorium bzw. die Souveränitätsrechte;

- zu Gesetzesentwürfen, die vom Staatspräsidenten bzw. vom Volksrat an den Konsultationsrat weitergeleitet werden;

- zu Fragen der allgemeinen Staatspolitik, der auswärtigen bzw. arabischen Politik.

Der Konsultationsrat ist verpflichtet, Stellung zu nehmen, mit Ausnahme der zuletzt genannten Fragen. Er hat die Stellungsnahme innerhalb eines Monats abzugeben, wobei die Frist um einen weiteren Monat verlängert werden kann. Gibt er während dieser Frist keine Stellungnahme ab, gilt sein Schweigen als Zustimmung.

Schließlich hat der Konsultationsrat eine einflußreiche Funktion im Pressewesen, das durch die Verfassungsänderung von 1980 als Volksgewalt bezeichnet und in Art. 206-211 geregelt wurde. Er übt nämlich die Eigentumsrechte des Staates an den Verlagen der sogenannten Nationalzeitungen aus. Zu den Nationalzeitungen zählen solche, deren Verlage vorher der Arabischen Sozialistischen Union gehörten oder vom Konsultationsrat selbst gegründet sind.

31 Vgl. al-Garida ar-Rasmiya Nr. 11 vom 16.3.1989. 


\section{Andere staatsrechtliche Änderungen}

\section{Neuorganisation der Gebietsverwaltung}

Für die lokale Verwaltung gilt neben Art. 161-163 der Verfassung vor allem das Gesetz Nr. 43/1979, das zuletzt durch die Gesetze Nr. 50/1981 und 145/198832 geändert wurde. Das Gesetz Nr. 43/1979 folgt insoweit seinem Vorgänger, als es die Lokalverwaltung, abweichend von der in anderen Staaten üblichen Regelung, auf zwei Arten von Räten verteilt: die gewählten lokalen Volksräte und die Exekutivräte. Erstere bestehen auf fünf Ebenen, nämlich als Räte der Gouvernorate, der Zentralstädte, der Städte, Stadtviertel und Dörfer. Die Mitglieder werden durch Listenwahl mit absoluter Mehrheit bestimmt. Nach dem Gesetz Nr. 145/1988 wird neuerdings ein einziger Sitz in jedem Volksrat durch Direktmandate besetzt. Die übrigen Sitze sind der Listenwahl vorbehalten. Diese Regelung wird kritisiert, da sie der unterschiedlichen Flächengröße und Wählerzahl der verschiedenen Vertretungsebenen nicht Rechnung trägt. Die Wahllisten werden von den politischen Parteien erstellt, wobei unter den vorgeschlagenen Kandidaten zumindest die Hälfte Bauem oder Arbeiter sein müssen. Angehörige verschiedener politischer Parteien dürfen nach dem Gesetz Nr. 145/1988 nicht auf einer Wahlliste stehen. Die bisher mit einem Sitz zwingend vorgeschriebene Beteiligung von Frauen wurde aufgehoben.

Der Exekutivrat besteht aus den Spitzenvertretern der Verwaltung auf jeder der fünf oben genannten Ebenen.

Das Verhältnis zwischen lokalem Volksrat und Exekutivrat ist dem zwischen Regierung (Exekutive) und Volksversammlung (Legislative) nachgebildet. Dies steht allerdings mit dem Wesen der lokalen Räte nicht in Einklang. Diese sind keine Volksvertretung im Sinne einer Legislative, sondern vielmehr Exekutivorgane mit Vollzugskompetenzen hinsichtich der Schaffung und Verwaltung lokaler Einrichtungen.

Der gewählte lokale Verwaltungsrat hat nach dem Gesetz Nr. 43/1979 ähnlich wie die Volksversammlung das Recht, einen Gegenstand zur Diskussion zu stellen oder Untersuchungen durchzuführen. Das durch Gesetz Nr. 50/1981 wieder eingeführte Interpellationsrecht sowie das Recht, dem Exekutivrat das Vertrauen zu entziehen, wurden durch das Gesetz 145/1988 aufgehoben.

Der Exekutivrat untersteht einer vielfältigen Aufsicht. Die Aufsicht steht dem Staatspräsidenten, dem Ministerrat, dem Ministerpräsidenten und dem jeweiligen Minister hinsichtlich der in sein Ressort fallenden Tätigkeiten der Gebietsverwaltung zu. Dem Gouverneur ist

32 Vgl. Sulayman at-Tammawi, al-Qanun raqam 145/1988 bi-isdar qanun al-hukm al-mahali, Magallat al-‘Ulum al-Idariya, 30. Jg. (Dezember 1988), S. 7 ff. 
eine generelle Aufsicht über die Einheiten der Gebietsverwaltung zuerkannt. Außerdem hat der Volksrat eine Aufsichtsbefugnis jeweils gegenüber den Volksräten der unteren Ebenen. Schließlich üben die Mitglieder der Volksversammlung (Legislative) auch ihre Aufsicht dadurch aus, daß sie zur Teilnahme an den Sitzungen der Volksräte ihrer Wahlbezirke mit einem Vorschlags- und Fragerecht, jedoch ohne Stimmrecht berechtigt sind. Das Gesetz Nr. 145/1988 hat auch den Mitgliedern des Konsultationsrates dieses Teilnahmerecht eingeräumt, allerdings beschränkt auf die Volksräte der Gouvemoratsebene.

Die oben dargelegte mehrfache Aufsicht ist in der Literatur kritisiert worden, da sie die Volksräte in der Ausübung ihrer Tätigkeit lähmt. Trotz dieser Kritik hat das Gesetz Nr. 145/198833 die Aufsichtsbefugnis der Zentralregierung verstärkt. Nach Art. 112 ff. kann der Ministerpräsident anordnen, daß sich Gouvemorate über bestimmte Maßnahmen abstimmen, wie die Schaffung von Dienstleistungen und gemeinschaftlichen Unternehmen; allerdings nur auf Vorlage des Ministers für Gebietsverwaltung und nach Einholung der Stellungnahme der zuständigen Gouvemeure. Außerdem kann der Ministerpräsident auf Vorlage des zuständigen Ministers für Gebietsverwaltung und nach Einholung der Zustimmung des zuständigen Gouverneurs eine Maßnahme anordnen, die von einem lokalen Volksrat gemäß dem Staatsplan, dem verabschiedeten Haushalt oder den Gesetzen hätte vorgenommen werden sollen, wenn für deren Unterlassung kein gesetzlich rechtfertigender Grund besteht und die Durchführung der Maßnahme vorher angemahnt worden war.

Bei Meinungsverschiedenheiten zwischen den Volksräten und den Aufsichtsstellen entscheidet die Zentralregierung, wobei zwischen dem Volksrat auf der Ebene des Gouvemorats und den Volksräten der übrigen Ebenen unterschieden wird. Der Gouverneur hat ein Vetorecht gegen die vom Volksrat des Gouvemorats gefaßten Beschlüsse. Besteht der Volksrat auf seinem Beschluß, dem der Gouvemeur widersprochen hat, so hat der für die Gebietsverwaltung zuständige Minister den Fall dem Ministerrat zur endgültigen Entscheidung vorzulegen. Über Meinungsverschiedenheiten hinsichtlich der Beschlüsse der Volksräte der unteren Ebenen entscheidet endgültig der für Gebietsverwaltungsfragen zuständige Minister.

Nach dem Gesetz Nr. 145/1988 vertritt der Gouverneur die Exekutive auf der Ebene des Gouvemorats. Seine Zuständigkeiten gegenüber den Verwaltungseinrichtungen und den selbständigen öffentlichrechtlichen juristischen Personen mit zentralem Charakter sind beschränkt. Der Gouvemeur ist gegenüber dem Ministerpräsidenten im Hinblick auf die Ausübung seiner gesetzlichen Zuständigkeiten verantwortlich, ihm aber nicht unterstellt. Die Einflußnahme des Ministerpräsidenten auf die Tätigkeiten der Gouverneure beschränkt sich auf Vorschläge an den Ministerpräsidenten, bestimmte Gouverneure zu emennen oder zu entlassen.

33 at-Tammawi (Fn. 32), S. $25 \mathrm{ff}$. 


\section{Neuorganisation der Judikative}

Die Hauptgrundsätze der richterlichen Gewalt finden sich in Art. 165-173 der Verfassung. Ausführliche Regelungen sind vor allem in den Gesetzen Nr. 46/1972 betreffend die richterliche Gewalt (Gerichtsverfassung), Nr. 47/1972 betreffend den Staatsrat (Conseil d'Etat) und Nr. 48/1979 über den Hohen Verfassungsgerichtshof enthalten34.

Gemäß Art. 1 des Gesetzes Nr. 47/1972 über die richterliche Gewalt gibt es ordentliche Gerichte auf vier Ebenen: den Kassationshof, die Appelationsgerichtshöfe, die Land- und Amtsgerichte. Organisation und Zusammensetzung der genannten Gerichte sind in den Artikeln 2-14 geregelt. Den ordentlichen Gerichten obliegt die allgemeine Zuständigkeit für Streitigkeiten mit Ausnahme von Verwaltungsstreitigkeiten, die in die Zuständigkeit des Staatsrates fallen (Art. 15). Die Nachprüfbarkeit von Hoheitsakten ist der Zuständigkeit der allgemeinen Gerichte entzogen (Art. 17). Das Gesetz enthält die Organisation der Staatsanwaltschaft und die Regelung ihrer Kompetenzen (Art. 21-27) sowie Vorschriften zur Emennung, Beförderung, Anciennetät, Versetzung, Abordnung, Unabsetzbarkeit und Entlohnung der Richter sowie über deren Pflichten (Art. 38-77). Regelungen über Beschwerden und Rechtsmittel gegen Beschlüsse in Angelegenheiten der Richter sowie Disziplinarverfahren gegen sie finden sich in Art. 79-115. Ferner enthält das Gesetz Bestimmungen über die Staatsanwaltschaft (Art. 116-134), die Richtergehilfen (Art. 134) und die Gerichtsangestellten (Art. 135-171)35.

\section{a) Einrichtung des Hohen Verfassungsgerichtshofes}

Eine eigenständige Verfassungsgerichtsbarkeit wurde in Ägypten erst durch das Gesetz Nr. 81/1969, also zwei Jahre vor der Proklamation der Verfassung von 1971, geschaffen. Diese sieht in den Artikeln 174-178 die Bildung eines Hohen Verfassungsgerichtshofes vor, der als eine selbständige autonome Gerichtsbarkeit bezeichnet wird (Art. 174). Er hat die ausschließliche Zuständigkeit, die Verfassungsmäßigkeit von Gesetzen und Verordnungen zu prüfen und gesetzliche Vorschriften zu interpretieren. Andere Zuständigkeiten, das Verfahren und die Zusammensetzung des Verfassungsgerichtshofes sollen durch Gesetz bestimmt werden (Art. 175-176).

Dieses Gesetz wurde erst am 29.8.1979 (Gesetz Nr. 48/1979) erlassen. Bis zur Zusarnmensetzung des neu geschaffenen Verfassungsgerichtshofes hat der seit 1969 bestehende "Hohe Gerichtshof" (al-mahkama al-'ulya) in Anwendung des Art. 192 der Verfassung von 1971

34 at-Tammawi (Fn. 2), S. $115 \mathrm{ff}$.; al-'Adala (Fn. 8) S. 102 ff., S. $115 \mathrm{ff}$.

35 al-'Adala (Fn. 8), S. 104 ff., $118 \mathrm{ff}$. 
die Zuständigkeiten ausgeübt, die denen des jetzigen Hohen Verfassungsgerichtshofes weitgehend entsprachen.

Der Hohe Verfassungsgerichtshof besteht aus einem Präsidenten sowie einer "genügenden" Anzahl von Mitgliedern. Die damit offengelassene Anzahl der Mitglieder wird von der Lehre weitgehend kritisiert, da die politische Macht über die Emennung weiterer Mitglieder die Unabhängigkeit des Gerichtshofes beeinträchtigen kann.

Zu den Voraussetzungen zur Emennung als Richter (Art. 4) zählt u.a. ein Mindestalter von 45 Lebensjahren, die Befähigung zum Richteramt und eine bestimmte Dauer der Berufsausübung entweder als Richter oder als Professor der Rechtswissenschaft an Universitäten. Mindestens zwei Drittel der Mitglieder des Hohen Verfassungsgerichtshofes müssen aus Mitgliedem bestehender Gerichte bestellt werden.

Die Richter des Hohen Verfassungsgerichtshofes sind unabsetzbar und dürfen nicht ohne ihr Einverständnis in andere Ämter versetzt werden.36

Die Zuständigkeit des Hohen Verfassungsgerichtshofes ist in Art. 25-26 des Gesetzes Nr. 48/1979 geregelt. Ihm sind neben den in der Verfassung enthaltenen (siehe oben) folgende Zuständigkeiten zugewiesen:

- Prüfung der Verfassungsmäßigkeit von Gesetzen und Rechtsverordnungen;

- Entscheidungen über positive bzw. negative Zuständigkeitskonflikte zwischen zwei Gerichtsbarkeiten (dies ist der Fall, wenn zwei Gerichte, die verschiedenen Gerichtsbarkeiten angehören, ihre Zuständigkeit in einem anhängigen Verfahren in derselben Sache bejahen bzw. verneinen);

- Entscheidungen über Streitigkeiten hinsichtlich der Vollstreckung zweier sich widersprechender rechtskräftiger Urteile verschiedener Gerichtsbarkeiten;

- Auslegung mit Gesetzeskraft der von der Legislative erlassenen Gesetze sowie der vom Staatspräsidenten nach der Verfassung verabschiedeten Beschlüsse, wenn Meinungsverschiedenheiten über ihre Auslegung bestehen, die so bedeutsam sind, daß die Vereinheitlichung ihrer Auslegung für geboten erachtet wird. Normalerweise steht die Auslegung von Vorschriften dem Gericht zu, das sie anwendet. Deshalb ist das Recht, das Hohe Verfassungsgericht wegen einer für erforderlich gehaltenen einheitlichen

36 Y. al-asch-Schamawi, Muqarana bain qanun al-Mahkma ad-dusturiya al-'ulya, Magallat al-'Ulum al-Idariya, 22. Jg. (Dezember 1980), S. 7 ff.; Sarif (Fn. 15), S. 83 ff.; at-Tammawi (Fn. 2), S. 118 ff.; al-'Adala (Fn. 8), S. 115 ff. 
Auslegung anzurufen, dem Justizminister auf Verlangen des Ministerpräsidenten, des Präsidenten der Volksversammlung oder des Hohen Rates des Richtertums vorbehalten. Die vom Hohen Verfassungsgerichtshof vorgenommene Auslegung hat Gesetzeskraft und muß im Gesetzesblatt veröffentlicht werden.

Das Verfahren37 zur Ủberprüfung der Verfassungsmäßigkeit von Gesetzen und Rechtsverordnungen ist zweigleisig ausgestaltet. Der erste Weg wird beschritten, wenn ein Gericht Bedenken an der Verfassungmäßigkeit einer Vorschrift in einem Gesetz oder einer Rechtsverordnung hat und diese Frage dem Verfassungsgerichtshof vorlegt. Ein zweiter Weg besteht darin, daß ein Gericht oder ein anderes Justizorgan das bei ihm anhängige Verfahren auf Antrag einer der Parteien aussetzt, um der Partei Gelegenheit zu geben, binnen einer Frist von drei Monaten eine Verfassungsklage zu erheben (Art. 29 Abs. 2 des Gesetzes Nr. 48/1979).

Die Entscheidungen des Hohen Verfassungsgerichtshofes 38 werden von sieben Mitgliedern gefällt (Art. 3). Sie sind endgültig; gegen sie kann kein Rechtsmittel eingelegt werden (Art. 48). Sie sind für alle Staatsgewalten bindend und werden innerhalb von 15 Tagen nach ihrer Verkündung im Gesetzblatt veröffentlicht. Erklärt der Hohe Verfassungsgerichtshof ein Gesetz bzw. eine Rechtsverordnung für verfassungswidrig, ist deren Anwendung ab dem Tag nach der Veröffentlichung der Entscheidung unzulässig. Die Entscheidungen haben keine Rückwirkung, es sei denn, es handele sich um eine Verurteilung auf Grund einer verfassungswidrigen Strafnorm. In diesem Falle gilt das Strafurteil als inexistent.

\section{b) Stärkung des Staatsrates (Conseil d'Etat)}

Art. 172 der Verfassung von 1971 ist dem 1946 eingerichteten Staatsrat gewidmet; über ihn handelt ausführlich das Gesetz Nr. 47/197239, das seither mehrmals geändert worden ist. Durch dieses Gesetz ist seine Stellung verstärkt worden, vor allem durch die Zuweisung neuer Zuständigkeiten auf Kosten der allgemeinen Gerichte.

Der Staatsrat ist eine selbständige Gerichtsbarkeit, die dem Justizminister untersteht. Er ist in drei Abteilungen gegliedert: die gerichtliche Abteilung, die Gutachtenabteilung und die Abteilung für Gesetzgebung.

- Die gerichtliche Abteilung des Staatsrates ist eine über mehrere Instanzen aufgebaute Verwaltungsgerichtsbarkeit und besteht aus dem Hohen Verwaltungsgerichtshof, den

37 Scharif(Fn. 15), S. $287 \mathrm{ff}$.

38 Scharif(Fn. 15), S. $452 \mathrm{ff}$.

39 at-Tammawi (Fn. 2), S. 116 ff;; N. 'Attiya, in: al-'Adala (Fn. 8), S. 128 ff. 
Verwaltungs- und den Disziplinargerichten und aus dem Korps der Kommissare (Art. 3). Aufgabe der Kommissare ist es, die Fälle zwecks Vorlage bei den Verwaltungsgerichten vorzubereiten. Der Hohe Verwaltungsgerichtshof, der an der Spitze der Verwaltungsgerichtsbarkeit steht, gliedert sich in Kammem (bestehend normalerweise aus fünf Richtem, für die Zulassung der eingelegten Rechtsmittel jedoch nur aus drei Richtem). Er hat ähnliche Funktionen wie der Kassationshof, unterscheidet sich aber von letzterem dadurch, daß seine Kontrollfunktion sich sowohl auf Tatsachen als auch auf die Gesetzesanwendung erstreckt. So hat er eine allgemeine Zuständigkeit für alle Verwaltungsstreitigkeiten mit Ausnahme derer, die in die enumerativ aufgezählten Zuständigkeiten der unteren Verwaltungsgerichte fallen. Außerdem ist er eine Berufungsinstanz für die Entscheidungen der Verwaltungsgerichte.

Die Disziplinargerichte sind zuständig für Disziplinarverfahren gegen Bedienstete des öffentlichen Dienstes und gegen andere Beschäftigte, sofern eine gesetzliche Zuständigkeitsverweisung besteht.

- Die Gutachtenabteilung besteht aus Referaten, die für bestimmte Sachgebiete zuständig und denen die entsprechenden Ministerien zugewiesen sind. Sie erteilt Rechtsauskünfte über ihr vorgelegte Fragen, gleichgültig, ob die Einholung der Rechtsauskunft zwingend oder fakultativ ist. Die Rechtsauskunft ist für den Fragesteller nicht bindend. In der Praxis wird allerdings aus Haftungsgründen selten von ihr abgewichen.

- Die Abteilung für Gesetzgebung besteht aus einem der Vizepräsidenten des Staatsrates sowie einer Anzahl von Richtem. Ihr sind außerdem Referendare (nuwwab) und Hilfsreferendare zugeordnet. Der Abteilung für Gesetzgebung obliegt es in erster Linie, die Formulierung der zu erlassenden Gesetze und Verordnungen zu prüfen; dementsprechend sind die Ministerien und sonstigen Behörden angewiesen, alle Entwürfe von Gesetzen, Verordnungen und Präsidialbeschlüsse mit Gesetzeskraft vorzulegen. Die Mißachtung dieses Gebots gilt nach der Verwaltungsrechtsprechung als Verfahrensfehler mit der Folge, daß der Beschluß als fehlerhaft angefochten werden kann. Handelt es sich um ein Gesetz, so gilt es als verfassungswidrig.

Der Staatsrat hat neben den drei Abteilungen eine Generalversammlung, die sich aus einem Vizepräsidenten des Staatsrates, aus den als Vorsitzenden der beiden Abteilungen bestimmten Vizepräsidenten des Staatsrates, den Mitgliedsrichtem der Abteilung für Gesetzgebung sowie den Referatsleitern der Gutachtenabteilung zusammensetzt.

Die Generalversammlung hat folgende Zuständigkeiten:

- Auskunftserteilung über die in Art. 66 des Gesetzes Nr. 47/1972 aufgezählten wichtigen Fragen völkerrechtlicher, verfassungsrechtlicher und gesetzlicher Art sowie über 
andere Rechtsangelegenheiten, die wegen ihrer Bedeutung vom Staatspräsidenten, dem Präsidenten der Legislative, dem Ministerpräsidenten, einem der Minister oder vom Präsidenten des Staatsrates der Generalversammlung vorgelegt worden sind;

- Rechtsauskünfte in Fragen, in denen eines der Referate der Abteilung für Gesetzgebung eine andere Rechtsauskunft erteilt als die, die von einem anderen Referat oder von der Generalversammlung bei der Abteilung vertreten wurde;

- Rechtsauskünfte in Fragen, die ihr einer der Ausschüsse der Gutachtenabteilung wegen ihrer Bedeutung zuleitet;

- Regelungen von Konflikten zwischen den Ministerien, den diesen nicht zugeordneten Behörden, Anstalten, öffentlichen Körperschaften oder den Institutionen der lokalen Verwaltung;

- Prüfung der Entwürfe von Gesetzen, Präsidialbeschlüssen mit Gesetzeskraft und Verordnungen, die ihr die Abteilung für Gesetzgebung vorlegt.

\section{c) Neuregelung bzw. Einsetzung von Ausnahmegerichten}

Die ägyptische Justiz kannte und kennt neben den besonderen Gerichten (z.B. Militärgerichte) Ausnahmegerichte40. Diese sind wie erstere für bestimmte Straftaten bzw. -täter zuständig, ihre Einsetzung ist jedoch durch das Vorliegen besonderer Umstände bedingt. Sie sind nicht an das normale Verfahren der ordentlichen Gerichte gebunden und können ganz oder teilweise aus nicht berufsmäßigen Richtern bestehen. Sieht man von früheren Ausnahmegerichten, wie dem Revolutionsgericht von 1953, ab, gibt es heute noch folgende Ausnahmegerichte:

\section{aa) Die Gerichte für Staatssicherheit}

Die Gerichte für Staatssicherheit (mahakem amn ad-dawla) wurden durch Präsidialbeschluß Nr. 162 von 1958 geschaffen; ihr Bestehen war zunächst von der Fortgeltung des Ausnahmezustandes abhängig und daher provisorisch, bis ihnen durch das Gesetz Nr. 105

40 Kritisch dazu A. Sawi, al-Wasit fi sarh qanun al-muraf'at al-madaniya, Kairo 1987, S. 39 ff. 
von $1980^{41}$ ein Dauerstatus verliehen wurde. Sie sind ausschließlich zuständig für Straftaten gemäß:

- Kapitel 1-4 des 2. Abschnitts des Strafgesetzbuches (Verbrechen und Vergehen betreffend die innere und äußere Staatssicherheit, die Bestechung, Munition sowie die Unterschlagung öffentlicher Güter bzw. gegen diese gerichtete Übergriffe: Art. 77-119 bis StGB);

- Gesetz Nr. 34/1972 über den Schutz der nationalen Einheit;

- Gesetz Nr. 2/1977 über den Schutz der Freiheit des Heimatlandes und des Bürgers;

- Gesetz Nr. 40/1977 und Nr. 36/1979 über die politischen Parteien;

- Dekretgesetz Nr. 95/1945 über die Lebensmittelrationierung;

- Dekretgesetz Nr. 163/1950 über die Preisfestsetzung und die Gewinnspannenbeschränkung sowie die dazu erlassenen Beschlüsse, sofern die zu verhängenden Strafen höher als Gefängnisstrafen sind.

Diese Gerichtsart ist zweistufig aufgebaut. Am Sitz jedes Amtsgerichts (mahkama goz'iya) ist ein Gericht für Staatssicherheit geschaffen. Es ist für die letztgenannte Gruppe von Fällen zuständig, wenn diese mit nicht mehr als einer Gefängnisstrafe geahndet werden können. Ferner fallen in seine Zuständigkeit auch die Vergehen des Gesetzes Nr. 49/1977 über die "Vermietung und Veräußerung von Räumen sowie die Beziehungen zwischen Vermietern und Mietern". Gegen die Urteile des Gerichts für Staatssicherheit kann eine Berufung vor der Kammer eingelegt werden, die für die Berufung gegen die in strafbaren Vergehen ergangenen Urteile zuständig ist.

In jeder Stadt, in der es ein Landgericht (mahkama ibtida'ya) gibt, ist ein Hoher Gerichtshof für die Staatssicherheit eingerichtet. Er besteht aus drei Richtern, die aus den Richtern am Appellationshof rekrutiert werden. Den Vorsitz hat einer von ihnen im Rang eines

41 N.'Attiya, Mahakim amn ad-dawla, in Magallat al-'Ulum al-Idariya, 23. Jg. (Dezember 1981), S. 7 ff.; Ahmed S. al-Chatib, Mahakem amn ad-dawla, al-Muhama Nr. 7/8 (Sept./Okt.) 1988, S. $31 \mathrm{ff}$.; as-Sayyid H. Karrat, Nazariyat Sultat al-harb, Diss. Ain Sams (Kairo) 1988, S. 99 ff.; al'Adala (Fn. 8), S. 120 ff. Neben den nach dem Gesetz Nr. 105/1980 ständigen Staatssicherheitsgerichten können besondere Staatssicherheitsgerichte während des nach dem Präsidialbeschluß Nr. 162/1958 (s. oben im Text) proklamierten Ausnahmezustandes eingesetzt werden. Diese können ausschließlich aus Militärrichtem bestehen, sind nicht an die StPO gebunden und ihre Urteile werden mit Bestätigung des Staatspräsidenten rechtskräftig; gegen sie kann kein Rechtsmittel eingelegt werden. Nach einer Aufassung kann man das Rechtsmittel der Kassation gegen sie einlegen (Karrat S. 99f). Den Ausnahmecharakter dieser Gerichte wurde vom Kassationsgerichtshof (vgl. z.B. Beschluß vom 24.5.1976, offizielle Entscheidungssammlung, 27. Jg., S. 538) und vom Verwaltungsgerichtshof (Beschluß 23.12.1986, Klage Nr. 6685, 40. Gerichtsjahr) betont. Thre Zuständigkeit ist daher restriktiv auszulegen. Der Ausnahmezustand wurde kurz nach seiner Aufhebung am 15.5.1980 wieder am 22.10.1981 nach der Ennordung Sadats proklamiert und wird bis heute jährlich verlängert. 
Appellationshofspräsidenten. Hinzukommen können gemäß einem Beschluß des Staatspräsidenten zwei Armeeoffiziere ab einem bestimmten Rang (' amid), die aus den Richtern der Militärgerichtsbarkeit rekrutiert werden. Die Urteile des Gerichtshofes sind grundsätzlich rechtskräftig. Gegen sie kann nur das Rechtsmittel der Kassation eingelegt oder beim Vorliegen bestimmter Gründe die Wiederaufnahme des Verfahrens beantragt werden.

Die Ausnahmegerichte haben über die ihnen vorgelegten Fälle unverzüglich zu entscheiden. Die Intervention eines Nebenklägers zur Geltendmachung von zivilrechtlichen Ansprüchen ist unzulässig.

\section{bb) Das Wertegericht}

Das Wertegericht (mahkamat al-qiyam)42, geregelt in Gesetz Nr. 95/1980 über "den Schutz der Werte vor Schande", ist für die in Art. 3 und 16 aufgeführten Vergehen und für Übertretungen anderer aufgelisteter Gesetze zuständig. Diese Vergehen werden vom sozialistischen Generalankläger vor Gericht geltend gemacht und können zur Aberkennung politischer und bürgerlicher Rechte der angeschuldigten Person führen. Außerdem ist das Wertegericht für Vermögenssequestration nach dem Gestz Nr. 34/1971 zuständig.

Folgende Vergehen lösen nach dem Gestz Nr. 95/198043 die "politische Verantwortlichkeit" des Beschuldigten aus:

- Verbreitung von Äußerungen, die gegen die Offenbarungsreligionen gerichtet sind;

- Anstiftung der Jugend, sich von den religiösen Werten oder der Treue zum Vaterland loszusagen;

- Veröffentlichung bzw. Verbreitung von unrichtigen oder sensationellen, dem nationalen Interesse schädlichen Nachrichten oder Gerüchten im Ausland.

Übertretungen folgender Gesetze können dazu führen, zur politischen Verantwortung gezogen zu werden:

- Gesetz Nr. 34/1972 über den "Schutz der nationalen Einheit";

- Präsidialbeschluß Nr. 2/1977, der als Folge der mit den Preiserhöhungen für Grundnahrungsmittel am 17./18.1.1977 im Zusammenhang stehenden Unruhen und Zerstörungen erlassen wurde;

- Gesetz Nr. 40/1977 nebst Änderungsgesetz Nr. 36/1979 über die politischen Parteien;

- Gesetz Nr. 33/1978 über den "Schutz der inneren Front und des sozialen Friedens".

42 Vgl. al-'Adala (Fn. 8), S. 479 ff.; Chalid A. Sarhan, Mahkamat al-qiyam, Kairo 1988, S. 63 ff., der verfassungsrechtliche Bedenken zu der Regelung dieses Gerichts hat (S. $117 \mathrm{ff}$.).

43 Sarhan (Fn. 42), S. 81 ff. 
Außerdem ist das Wertegericht dafür zuständig, die Sequestration über das Vermögen von Personen, deren Ehegatten und Kinder nach dem Gesetz Nr. 34/1971 auf Antrag des sozialistischen Generalanklägers anzuordnen. Diese Maßnahme kann in folgenden Fällen ergehen:

a) Zum Schutz der Gesellschaft vor gefährlichen Personen, gegen die begründeter Verdacht auf Begehung von Taten besteht, die der Staatssicherheit, den wirtschaftlichen Interessen des sozialistischen Systems, dem politischen Leben oder der nationalen Einheit schädlich sind.

b) Vorliegen triftiger Indizien für persönliche Bereicherung aufgrund folgender Tatbestände:

- Mißbrauch von Ämtem, z.B. Mitgliedschaft in der Volksvertretung;

- Arglist, Konspiration oder Bestechung bei der Durchführung von Werkverträgen, Lieferungsverträgen oder sonstigen Verwaltungsverträgen mit der Regierung, mit Anstalten, mit Holdingorganisationen bzw. den letzteren unterstellten Gesellschaften des öffentlichen Sektors oder sonstigen juristischen Personen des öffentlichen Rechts;

- Schmuggel und Handel mit Rauschgift;

- Handel mit verbotenen Waren oder Schwarzmarkthandel mit Grundnahrungsmitteln sowie Arzneimitteln; .

- unrechtmäßige Aneignung von Sachen des öffentlichen Rechts oder solchen, die Privateigentum des Staates bzw. juristischer Personen sind.

Der Generalankläger kann den Arrest der unter a) aufgeführten Personen an einem sicheren Ort anordnen, wobei er diese Maßnahme innerhalb von 60 Tagen dem Wertegericht vorlegen muß. Das Gericht kann die Fortsetzung des Arrests für eine Höchstdauer von einem Jahr anordnen und ihn bis zu insgesamt fünf Jahren erneuem. 44

Das Wertegericht setzt sich aus sieben Mitgliedern zusammen. Der Vorsitz wird von einem der Vizepräsidenten des Kassationshofes wahrgenommen. Drei der Gerichtsmitglieder werden aus den Richtern am Kassationshof oder an den Appellationshöfen rekrutiert. Die drei anderen Mitglieder sind aus einer Liste von Persönlichkeiten des öffentlichen Lebens für zwei Jahre zu bestimmen. Letztere werden bei Antritt ihrer Tätigkeit vereidigt.

Der Hohe Wertegerichtshof ist als Berufungsinstanz bestimmt; er besteht aus neun Mitgliedern. Vier von ihnen werden aus den Richtern am Kassationshof oder an den Appellationshöfen bestimmt, die anderen vier Mitglieder aus einer Liste von Persönlichkeiten des öffentlichen Lebens. Ein Vizepräsident des Kassationshofes übernimmt den Vorsitz. 
Gegen die Urteile des Wertegerichts kann entweder der sozialistische Generalankläger oder der Verurteilte Rechtsmittel beim Hohen Wertegerichtshof einlegen. Wird das Rechtsmittel von ersterem eingelegt, so kann das Gericht das Urteil der unteren Instanz bestätigen, aufheben oder zugunsten bzw. Ungunsten des Verurteilten abändem. Eine Verschärfung der verhängten Maßnahme oder die Aufhebung des Urteils durch Freispruch darf aber nur auf Grund eines einstimmigen Votums der Gerichtsmitglieder ergehen.

Wird das Rechtsmittel vom Verurteilten eingelegt, so kann der Gerichtshof das Urteil nur bestätigen, aufheben oder zu seinen Gunsten abändern.

Die Einlegung des Rechtsmittels hat in der Regel nicht die Einstellung des Urteilsvollzugs zur Folge. Das Gericht kann dies allerdings auf Antrag anordnen, wenn andernfalls nicht mehr abwendbare rechtliche Schäden zu befürchten sind. Der Beschluß des Hohen Wertegerichtshofes ist rechtskräftig. Gegen ihn können keine weiteren Rechtsmittel eingelegt werden. Eine Wiederaufnahme des Verfahrens kann gegen rechtskräftig gewordene Urteile des Wertegerichts in folgenden Fällen beantragt werden:

- in zwei voneinander unabhängigen Verfahren ergangene Verurteilungen zweier verschiedener Personen in derselben Sache, zwischen denen ein Widerspruch besteht, aus dem sich die Unschuld eines der Verurteilten folgem läßt;

- Verurteilung eines der Zeugen oder Sachverständigen durch ein Strafgericht wegen falscher Zeugenaussage oder wegen Fälschung einer Urkunde, die im Verfahren vor dem Wertegericht vorgelegt wurde, wenn die Zeugenaussage, der Bericht des Sachverständigen oder die Urkunde im Urteil des Wertegerichts Bedeutung erlangt hat;

- auf Grund von Tatsachen oder Urkunden, die erst nach Abschluß des Verfahrens bekannt geworden sind, wenn sie die Unschuld des Verurteilten beweisen.

Der Antrag auf Wiederaufnahme des Verfahrens kann vom sozialistischen Generalanwalt, vom Verurteilten oder im Falle seines Todes von dessen Verwandten oder dessen Frau gestellt werden. Wird von einem Strafgericht ein Freispruch auf Grund der Unrichtigkeit der vorgeworfenen Tatsachen oder der Straflosigkeit einer der nach Art. 3 des Gesetzes Nr. 95/1980 mit der Aberkennung politischer und bürgerlicher Rechte sanktionierten Taten erlassen, so hat der sozialistische Generalankläger die Fortsetzung der von ihm durchgeführten Untersuchungsverfahren in derselben Sache einzustellen. Das Urteil des Strafgerichts hat Rechtskraft auch gegenüber dem Wertegericht, wenn kein rechtskräftiges Urteil von letzterem in derselben Sache erlassen wurde. Ist nach einer rechtskräftigen Verurteilung durch das Wertegericht beim zuständigen Strafgericht in derselben Sache auf Grund der Unrichtigkeit der vorgeworfenen Tatsachen oder der Straflosigkeit der Tat ein rechtskräftiges Urteil mit Freispruch ergangen, so kann der Verurteilte sich wegen der Verurteilung beim Staatspräsidenten beschweren, um eine Begnadigung von der verhängten Maßnahme zu erwirken. 
Hinsichtlich des Verfahrens vor dem Wertegericht gelten die im Gesetz Nr. 95/1980 über "den Schutz der Werte vor Schande" dazu bestimmten Vorschriften (Art. 35-38) und die Bestimmungen des Zivil- und Handelsprozeßgesetzes, des Beweisgesetzes und des Strafprozeßgesetzes, sofern sie mit ersterem in Einklang stehen. Die Intervention eines Nebenklägers zur Geltendmachung von zivilrechtlichen Ansprüchen ist vor dem Wertegericht unzulässig. Im Verfahren vor dem Wertegericht herrscht Anwaltszwang. Vor dem Kassationshof zugelassene Anwälte dürfen vor dem Wertegericht auftreten. Rechtsmittel gegen Versäumnisurteile des Wertegerichts sind unzulässig.

\section{Neuregelungen des Staatsangehörigkeitsrechts}

Sowohl das Gesetz Nr. 82 vom 22.6.1958 als auch sein Vorläufer vom 20.10.1956 wurden als Reaktion auf die politischen Umstände erlassen, nämlich die Suezkrise und den durch sie ausgelösten Krieg und vor allem auf die Vereinigung Ägyptens und Syriens zur Vereinigten Arabischen Republik. Obgleich Syrien aus dieser staatlichen Vereinigung 1961 ausschied und die Staatsangehörigkeit seiner eigenen Bevölkerung im selben Jahr und später 1969 regelte, blieb in Ägypten das Gesetz Nr. 82/1958 in Kraft. Die Rechtsprechung des Verwaltungsgerichts in Ägypten beschränkte allerdings die Geltung des Gesetzes auf die ägyptischen Staatsangehörigen und betrachtete die Syrer ab 1961 wieder als Auslän$\operatorname{der} 45$.

Erst in der Verfassung von 1971 wurde die Staatsangehörigkeit, wie früher, wieder als ägyptisch bezeichnet. Einzelheiten sollten durch Gesetz geregelt werden (Art. 6). Dies geschah erst durch das Gesetz Nr. 26 vom 21.5.1975, das das bis dahin geltende Gesetz von 1958 ablöste. Es berücksichtigte die nach dem Austritt Syriens geschaffene politische Lage und machte dem wirklichkeitswidrigen Rechtsszustand ein Ende.

Folgende Hauptlinien durchziehen das Gesetz Nr. 26/197546:

- Wiederbelebung der originären ägyptischen Staatsangehörigkeit (nationalité originaire) durch Anknüpfung an die vor der Ausrufung der Vereinigten Arabischen Republik erlassene gesetzliche Regelung. Die originäre Staatsangehörigkeit ist diejenige, die der Staat bei seiner Gründung zur Bestimmung seiner Bevölkerung festgelegt hat. Von ihr wird in der Regel der Erwerb der Staatsangehörigkeit kraft Geburt abgeleitet.

45 Beschluß vom 28.12.1965 (Klage Nr. 59, 20. Gerichtsjahr), dazu 'Izz ad-Din 'Abd Allah, alQanun ad-dawli al-chas, 11. Aufl., Kairo 1986, Bd. 1, S. 364.

46 Fouad Riad, in: Juris-Classeur, Nationalité: sub Egypte (Stand 11, 1985) Nr. 39 ff. 
Dabei wurde allein an dem Erwerb jure sanguinis a patre festgehalten. Der Erwerb jure sanguinis a matre hat bis jetzt trotz zunehmender Kritik der Lehre keine Aufnahme gefunden. Die Abstammung von einer ägyptischen Mutter kann die ägyptische Staatsangehörigkeit nur dann vermitteln, wenn zusätzliche Voraussetzungen vorliegen:

- besondere Regelung der während des Zusammenschlusses Syriens und Ägyptens (1958-1961) entstandenen und für die Zuerkennung der ägyptischen Staatsangehörigkeit relevanten Sachverhalte;

- Lösungen für in der Praxis früherer ägyptischer Staatsangehörigkeitsgesetze ungenügend geregelter Sachverhalte, wie z.B. die Staatsangehörigkeit mit Ägyptern verheirateter Ausländerinnen, Verlust und Aberkennung der Staatsanghörigkeit, Förderung der Wiedererlangung der ägyptischen Staatsangehörigkeit nach deren Verlust als Folge des Erwerbs einer ausländischen Staatsangehörigkeit;

- Erschwerung der Einbürgerung durch die Forderung strenger Voraussetzungen, vor allem einer ziemlich langen Aufenthaltsdauer (mindestens $10 \mathrm{Jahre}$ ) und der Einräumung eines Ermessens der Exekutive, selbst beim Vorliegen der erforderlichen Voraussetzungen. Grund für diese Strenge ist die drückende Frage der Überbevölkerung;

- Bemühungen, der Staatenlosigkeit im Bereich des Erwerbs der Staatsangehörigkeit jure sanguinis, der Einbürgerung und des Verlustes der Staatsangehörigkeit Einhalt zu gebieten. So wird der Erwerb jure soli im Falle der Abstammung von unbekannten Eltern zugelassen. Außerdem wird für den Einbürgerungsantrag kein vorausgehender Verzicht auf die Staatsangehörigkeit des Antragstellers verlangt. Der Verlust der ägyptischen Staatsangehörigkeit wegen ausländischer Einbürgerung hängt von dem effektiven Erwerb der neuen Staatsangehörigkeit ab. Schließlich ist die Aberkennung der Staatsangehörigkeit im Unterschied zu den Gesetzen von 1956 und 1958 auf Fälle von offensichtlicher Illoyalität beschränkt worden. Die Befugnis zur Aberkennung steht nicht mehr dem Innenminister, sondern dem Ministerrat zu;

- Erleichterung des Nachweises der Staatsangehörigkeit mittels materiellrechtlicher Vorschriften, da die notwendige lückenlose Beurkundung von Personenstandsvorgängen vielerorts nicht eingehalten worden war.

Das Gesetz Nr. 26/1975 folgte weitgehend den Regelungen seines Vorgängers von 1958. Die Überbevölkerung zwang jedoch nach wie vor dazu, sowohl den Erwerb der Staatsangehörigkeit kraft Abstammung an das ius sanguinis a patre zu knüpfen, als auch die Voraussetzungen für die Einbürgerung restriktiv festzulegen. Die Vorschriften für den Erwerb der Staatsangehörigkeit nach dem ius sangiuinis und dem in einem bestimmten Ausnahmefall zuerkannten ius soli finden sich in Art. 2-3, die Einbürgerung in Art. 4, deren 
Wirkungen auf die Ehefrauen, die minderjährigen Kinder und den Eingebürgerten selbst in Art. 9, die Staatsangehörigkeit der mit Ägyptem verheirateten Ausländerinnen in Art. 7, der Verlust der Staatsangehörigkeit infolge des Erwerbs einer ausländischen Staatsangehörigkeit in Art. 10 bzw. die Heirat einer Ägypterin mit einem Ausländer in Art. 12, die Wiedererlangung der ägyptischen Staatsangehörigkeit nach dem Verlust, ihr Wiedererwerb nach dem Verlust infolge einer ausländischen Einbürgerung in Art. 15, die Aberkennung der ägyptischen Staatsangehörigkeit und der Widerruf der Einbürgerung in Art. 16, der Erwerb der Staatsangehörigkeit nach deren Aberkennung oder deren Widerruf in Art. 18.

Vorschriften über die Rückwirkung des Staatsangehörigkeitserwerbs, -widerrufs, -verlusts und -wiedererwerbs sind in Art. 19 enthalten.

Nach dieser Bestimmung sind dafür eine ausdrückliche Anordnung sowie eine gesetzliche Grundlage erforderlich. Zuständig als Empfänger von Erklärungen, Beschlüssen und Anträgen betreffend die Staatsangehörigkeit ist der Innenminister und der von ihm benannte Vertreter (Art. 20). Eine kollisionsrechtliche Vorschrift ist in Art. 23 enthalten, nach der das Volljährigkeitsalter gemäß dem ägyptischen Recht zu beurteilen ist. Zum Schluß folgen Vorschriften, die die Beweislast beim Geltendmachen bzw. Bestreiten der Staatsangehörigkeit (Art. 24), das Erfordemis einer öffentlichen Urkunde zum Nachweis der Eheschließung als relevante Tatbestandsvoraussetzung zum Erwerb bzw. Verlust der Staatsangehörigkeit (Art. 25) und den Vorrang internationaler Abkommen und Vereinbarungen über Staatsangehörigkeit,in denen Ägypten Vertragspartei ist und die materiellrechtliche Vorschriften enthalten (Art. 26), betreffen.

In Gesetz Nr. 26/1975 finden sich aber auch einige Unterschiede zum Gesetz Nr. 82/1958, wie z.B.:

1) Der Kreis der ägyptischen Staatsangehörigen zum Zeitpunkt des Inkrafttretens umfaßt folgende drei Gruppen:

- Personen, die keine Staatsangehörigkeit ausländischer Staaten besitzen, die vor dem 5. November 1914 in Ägypten ansässig waren und dort ihren Aufenthalt bis zum Inkrafttreten des Gesetzes beibehalten haben. Dabei wird die Aufenthaltsdauer der Aszendenten bzw. der Ehemänner auf die der Deszendenten bzw. der Ehefrauen angerechnet.

- Die jenigen, die am 22. Februar 1958, dem Zeitpunkt der Proklamierung der Vereinigten Arabischen Republik, die ägyptische Staatsangehörigkeit gemäß den Vorschriften des einschlägigen Gesetzes Nr. 391/1956 besaßen. 
- Diejenigen, die die Staatsangehörigkeit der Vereinigten Arabischen Republik gemäß den Vorschriften des entsprechenden Gesetzes Nr. 82/1958 aus einem der in Art. 1 aufgeführten drei Gründe erworben haben. Dabei werden als Voraussetzung starke Bindungen zu Ägypten verlangt. Streitfälle können allerdings wegen der verstrichenen Zeit kaum mehr auftreten.

2) Ein Kind, das von einer ägpytischen Mutter im Ausland geboren und dessen Vater unbekannt, unbekannter Staatsangehörigkeit oder staatenlos ist, kann innerhalb eines Jahres nach seiner Volljährigkeit für die ägyptische Staatsangehörigkeit optieren, selbst wenn es nicht ehelich ist. Außerdem wurde der bisher vor der Volljährigkeit erforderliche fünfjährige Aufenthalt in Ägypten durch einen gewöhnlichen Aufenthalt im Zeitpunkt der Zustellung der Optionserklärung ersetzt (Art. 3).

3) Eine Einbürgerung erfolgt generell durch Beschluß des Innenministers (Art. 4). Handelt es sich aber um das Oberhaupt einer Religionsgemeinschaft oder um einen Antragsteller, der sich um Ägypten besonders verdient gemacht hat, so kann er durch Beschluß des Staatspräsidenten ohne die Voraussetzungen des Art. 4 eingebürgert werden (Art. 5). Für einen Ausländer gilt eine erleichterte Einbürgerung, wenn er und sein Vater in Ägypten geboren sind und wenn dieser der arabischsprachigen oder islamischen Bevölkerungsmehrheit eines fremden Landes angehört. In diesem Falle reicht eine Jahresfrist ab Erreichen der Volljährigkeit zur Stellung des Einbürgerungsantrages aus (Art. 4 Ziff. 3); für andere Bewerber, die in Ägypten geboren sind und dort bei Volljährigkeit ihren gewöhnlichen Aufenthalt haben (Ziff. 4), ist der Antrag binnen Jahresfrist nach Erreichen der Volljährigkeit zu stellen. In beiden Fällen sind die üblichen Leumunds- und Integrationsvoraussetzungen nötig.

4) Die Beendigung der Ehe durch den Tod des ägyptischen Ehemannes steht der Einbürgerung seiner ausländischen Frau nicht mehr im Wege, wenn der Einbürgerungsantrag zu seinen Lebzeiten gestellt wurde und die vorgeschriebene Zweijahresfrist nach der Antragstellung abgelaufen ist.

5) Das bisherige Recht eines minderjährigen Kindes, das kraft Gesetzes in die Einbürgerung seines Vaters einbezogen worden war, mit Erreichen seiner Volljährigkeit für die ursprüngliche Staatsangehörigkeit des Vaters zu optieren, ist beibehalten; der Verlust seiner ägyptischen Staatsangehörigkeit erfolgt allerdings erst nach dem Erwerb der optierten Staatsangehörigkeit.

Zur Ausübung seiner politischen Rechte ist es jedoch erst nach Ablauf einer bestimmten Zeitspanne befugt; hiervon kann es durch Entscheid des Staatspräsidenten befreit werden. Andere, den Ägyptern vorbehaltene Rechte, wie z.B. Emennung zum Beamten, unterliegen nicht mehr der Voraussetzung eines Zeitablaufs. 
6) Der Erwerb einer fremden Staatsangehörigkeit im Ausland führt nur dann zum Erlöschen der ägyptischen Staatsangehörigkeit, wenn dieser vom Innenminister genehmigt worden ist (Art. 10 Abs. 1). Daneben gibt es die Möglichkeit, den Erwerb der fremden Staatsangehörigkeit zu gestatten, ohne daß die ägyptische verlorengeht (Art. 10 Abs. 3)47; ferner - wie bisher - die ägyptische bei nicht genehmigtem Erwerb der fremden Staatsangehörigkeit als Sanktion abzuerkennen (Art. 16 Nr. 1).

7) Eine ägyptische Ehefrau behält jetzt ihre Staatsangehörigkeit bei, wenn ihr ägyptischer Ehemann eine ausländische Staatsangehörigkeit annimmt, es sei denn, sie entscheidet sich für die neue Staatsangehörigkeit des Ehemannes und erwirbt sie nach dem maßgeblichen ausländischen Recht. Sie kann jetzt aber nach den Regeln des Art. 10 Abs. 3 die ägyptische Staatsangehörigkeit behalten.

8) Eine ägyptische Staatsangehörige, die eine nach dem Heimatrecht des Ehemannes gültige, aber nach ägyptischem Recht nichtige Ehe geschlossen hat, bleibt Ägypterin (z.B. Heirat einer Muslimin mit einem Nichtmuslim im Ausland). Der Innenminister kann ihr aber die Staatsangehörigkeit aberkennen, wenn sie die Staatsangehörigkeit ihres Mannes erworben hat (Art. 12 Abs. 2).

9) Eine Frau, die ursprünglich die ägyptische Staatsangehörigkeit besaß, aber diese verloren hat, oder die ägyptischer Herkunft ist, erwirbt die ägyptische Staatsangehörigkeit auf Antrag, sobald diese ihrem Mann verliehen ist bzw. sie mit einem Ägypter die Ehe schließt (Art. 14).

10) Die mittels Täuschung bzw. unrichtiger Aussage erworbene Staatsangehörigkeit kann nicht mehr unbegrenzt, sondern nur innerhalb von 10 Jahren nach dem Staatsangehörigkeitserwerb entzogen werden (Art. 15 Abs. 1).

11) Die Aberkennung der Staatsangehörigkeit erfolgt nicht mehr durch den bisher vorgesehenen Beschluß des Staatspräsidenten, sondern durch einen begründeten Beschluß des Ministerrats (Art. 16). Ferner sind die Aufnahme eines Arbeitsverhältnisses bei einer ausländischen Stelle ohne vorherige Genehmigung oder die endgültige Auswanderung keine Aberkennungsgründe mehr. Die in das neue Gesetz übernommenen Gründe sind

47 Diese Regelung wird durch den Wunsch vieler Emigranten, die Bindung an ihr Herkunftsland aufrechtzuerhalten, gerechffertigt. Dadurch wird die Doppelstaatsangehörigkeit entgegen der Tendenz vieler ausländischer Rechte, diese Erscheinung zu bekämpfen, gefördert. Art. 10 des Gesetzes Nr. 110/1983 betreffend die Emigration bekräftigt Art. 10 Abs. 3 des ägyptischen Staatsangehörigkeitsgesetzes, indem er dem ägyptischen Emigranten die Einbürgerung im Gastland unter Beibehaltung seiner ägyptischen Staatsangehörigkeit erlaubt. Kritisch zum ägyptischen Recht Ibrahim A. Ibrahim, Talafi izdiwag al-ginsiya, Kairo 1985, S. 112 ff;; anderer Auffassung Hischam Sadiq, al-Ginsiya, Alexandria 1977, S. 493. 
z.B. Kriegsdienst im Ausland, Verbrechen gegen die Staatssicherheit oder Mitgliedschaft in einer ausländischen umstürzlerischen Organisation.

\section{Zusammenfassung und Ausblick}

Die Verfassung von 1971 unterscheidet sich in ihren Grundsätzen kaum von ihrer Vorgängerin. Sie hat kaum etwas an der Ideologie, der sozio-ökonomischen Basis und der Form des bestehenden politischen Systems geändert, das durch die Einheitspartei, das Einkammer-System, die Volksvertretung und die übergewichtige Stellung des Staatspräsidenten und der ihm unterstehenden Regierung gekennzeichnet ist.

Die Verfassungsänderung von 1980 brachte einige wichtige Emeuerungen, vor allem die Schaffung des Konsultationsrats und die nachträgliche verfassungsrechtliche Verankerung des schon 1976 zugelassenen Mehrparteiensystems. Die erhoffte Stärkung der Legislative ist aber größtenteils ausgeblieben. Dem Konsultationsrat wurden lediglich beratende, aber keine legislativen Befugnisse zuerkannt. Die Inkonsistenz des Verfassungssystems, aber auch die Eingriffe vor allem Sadats haben zu einer dauerhaften Schwächung des Mehrparteiensystems geführt.

Ein Ausweg aus der Krise des Mehrparteiensystems in Ägypten hängt von der Einigkeit über die Ursachen der Krise in der Struktur des politischen Systems selbst ab48. Die Struktur der politischen Institutionen hat sich sowohl der Form als auch der Tätigkeit nach seit der Revolution von 1953 kaum geändert, obgleich die ihr zugrundeliegende wirtschaftliche und soziale Struktur, sowohl hinsichtlich ihrer eigenen Institutionen als auch der einschlägigen Regeln für die Wechselwirkungen zwischen den verschiedenen sozialen Gruppen immensen Wandlungen unterzogen wurden. Das Regierungssystem der Ära Nassers weist trotz seiner Mängel einen Einklang zwischen einerseits der politischen Struktur, die totalitär und sozialistisch orientiert war, und andererseits der ihr zugrundeliegenden wirtschaftlichen und sozialen Struktur mit einer Zentralplanung und einer allumfassenden Staatskontrolle der sozio-ökonomischen Wechselwirkung unter den verschiedenen sozialen Gruppen auf.

Der genannte Einklang bestand schon nicht mehr in der Ära Sadat und ist bis heute ausgeblieben. Während die autoritär und totalitär orientierte Struktur des politischen Systems weiterbesteht, hat die ihr zugrundeliegende sozio-ökonomische Struktur durch den Ubergang zu einer ungehemmten Wirtschaftsliberalität und der Zulassung der Marktmechanismen eine gravierende Änderung erfahren. Diese hat zur Entstehung neuer sozialer

Vgl. zu folgenden Ausführungen Nafa (Fn. 24), S. 61 ff. 
und politischer Gruppen geführt, deren Assimilation den bestehenden politischen Institutionen nicht gelungen ist.

Unter drei Alternativen, nämlich der Zulassung eines vollständigen politischen Liberalismus, einer Rückkehr zur Zentralplanung und allumfassenden Staatskontrolle der sozioökonomischen Wechselwirkung unter den gesellschaftlichen Gruppen und schließlich einer wirtschaftlichen, sozialen und politischen Reform hat letztere die größere Aussicht auf eine Wiederherstellung des vermißten Einklangs. Dies erfordert aber:

- ein soziales Programm, das von allen Gruppen einverständlich oder zumindest mehrheitlich erarbeitet werden sollte,

- die Ưbergabe des Vorsitzes der Regierungspartei an eine befähigte Persönlichkeit,

- die Einführung der Freiheit zur Gründung neuer politischer Parteien unter Beachtung sachlicher Kriterien,

- den Erlaß eines neuen Wahlgesetzes mit einer nicht überhöhten Sperrklausel sowie einer Chancengleichheit sowohl für Direktmandate als auch Parteienlisten,

- die Aufhebung der oben genannten, von Sadat erlassenen Sondergesetze sowie des Ausnahmezustandsgesetzes Nr. 162/1958, da die Verfassung dem Staatspräsidenten ausreichende Befugnisse zum Erlaß von Notstands- und Ausnahmezustandsgesetzen zuerkennt. 


\section{Errata}

Im Aufsatz "Obberblick über die Entwicklung des ägyptischen Staatsrechts seit den 70er Jahren" (VRU 1990, Heft 3, S. 297 ff.) finden sich in den Seiten zwischen 298 und 308 einige Fehler. Diese werden wie folgt berichtigt:

S. 298 Text Zeile 3: statt "Die Volksbefragung": "Der Volksentscheid".

Text Zeile 15: statt "aufzukommen (Art. 56)5": "aufzukommen (Art. 57)5".

Fn. 3 Zeile 1: statt "Volksbefragung": "Volksentscheide".

S. 302 Text Zeile 15: Fußnotennummer streichen.

Fn. 18

gehört auf S. 303.

S. 303 Text: $\quad$ Fußnotennummem 19, 2021 ersetzen durch 18, 19, 20.

S. 304 Text Zeile 9: statt "abgelehnt": "abgelehnt"21.

Text letze Zeile: Fußnotennummer streichen.

Fn. 22 Zeile 2: statt "21 ": "20".

Zeile 5: statt "Hidr": "Chidr".

Fn. 23: $\quad$ wird zu Fn. 26 auf S. 307.

S. 305 Text u. Fußnote: Fußnotennummer 24 ersetzen durch 23.

S. 306 Text u. Fußnoten: Fußnotennummer 25 und 26 ersetzen durch 24 und 25 .

S. 307 Text Zeile 8: Fußnotennummer 27 ersetzen durch 26 .

Text Zeile 13: statt "geregelt.": "geregelt27.".

Fußnoten: hinter Fn. 25 (geändert aus 26):

übernehme Fn. 26 (geändert aus 23) von S. 304/305.

S. 308 Fn. 28 Zeile 9: statt "hatte": "wurde".

Fn. 29 Zeile 1: statt "Haild": "Chalid".

Zeile 2: statt Fußnotennummer "25": "28".

Zeile 5: statt Fußnotennummer "25": "28".

Zeile 3 und vorletzte Zeile: statt "Art. 5b": "Art. 5 bis".

S. 313 Fn. 36 Zeile 1: statt "al-asch-Schamawi": "al-'Aschmawi".

Zeile 2: statt "Sarif": "Scharif".

S. 317 Fn. 41 Zeile 3: statt "Ain Sams": "Ain Schams". 